\title{
Annunci Congressi
}

\section{Genome and Envirome:}

\section{Roles and Interaction in Psychiatric Epidemiology}

The VIII Congress of the International Federation of Psychiatric Epidemiology (IFPE) will be held under this title at the International Conference Centre of the Academia Sinica in Taipei, Taiwan, 6-9 March 1999.

The Scientific programme will cover, in addition to the title theme, international collaborative research on schizophrenia, quality-of-life research and service evaluation, epidemiology of mental illness in primary care, epidemiology of mental illness in Asian Countries, and other topics. Half-day workshops will be held on research methods in genetic epidemiology, and on standardized clinical assessment using the «SCAN» instruments. The official languages of the Congress will be English and Chinese, with simultaneous translation in all plenary sessions.

For further details, please contact Professor Andrew Cheng, Division of Epidemiology, Institute of Biomedical Sciences (IBMS), Academia Sinica, Taipei, Taiwan (Phone 886.2.2789.9119, Fax 886.2.2785.3569 or 886.2.2782.3047, E-mail IFPE1999@gate.sinica.edu.tw, homepage http://www.sinica.edu.tw/-ifpe 1999/) 